\title{
Research Progress on Precipitation Accuracy Verification and Statistical Post-Processing in Ensemble Numerical Forecast System
}

\author{
Lingjie LI ${ }^{\mathrm{a}}$, Yongwei GAI ${ }^{\mathrm{b}}$, Leizhi WANG ${ }^{\mathrm{a}, 1}$, Liping LI ${ }^{\mathrm{c}}$, Xiaotian $\mathrm{LI}^{\mathrm{d}}$ and Rongjin \\ $\mathrm{ZHU}^{\mathrm{a}}$ \\ ${ }^{a}$ State Key Laboratory of Hydrology, Water Resources and Hydraulic Engineering \& \\ Science, Nanjing Hydraulic Research Institute, Nanjing, Jiangsu, China \\ ${ }^{\mathrm{b}}$ Water Resources Service Center of Jiangsu Province, Nanjing, Jiangsu, China \\ ${ }^{\mathrm{c}}$ Lvliang Meteorology Bureau of Shanxi Province, Lishi, Shanxi, China \\ ${ }^{\mathrm{d}}$ Water Resources Management Center, Ministry of Water Resources, Beijing, China
}

\begin{abstract}
The temporal and spatial accuracy of precipitation of ensemble numerical forecast systems is an important factor that affects the level of meteorological and hydrological coupled forecasting. This article focuses on the current research of verification of precipitation accuracy and statistical post-processing. The verification of forecast precipitation accuracy mainly focuses on the probabilistic characteristics such deterministic accuracy, the resolution, the forecasting skills and the degree of dispersion. Some mainstream statistical post-processing methods have strong performance of spatial downscaling and error correction, but they commonly have the defect of destroying the temporal and spatial dependent structure of precipitation. A comprehensive statistical post-processing method integrated the three functions is the development direction in the future. At the same time, statistical post-processing methods to improve the certainty and probabilistic accuracy of forecast precipitation need to be systematically identified. Its impact on the spatio-temporal dependence structure also needs to be improved.
\end{abstract}

Keywords. Ensemble numerical forecast, precipitation accuracy verification, statistical post-processing

\section{Introduction}

Ever since a long time, the hydrological forecasting features of the extending of effective foresight period, the improvement of forecasting accuracy and the quantifying of forecast uncertainty [1]. In recent years, the development of atmospheric dynamics and computer science has promoted the continuous advancement of numerical weather forecast technology. The global ensemble numerical forecast model can provide precipitation forecast information with a longer forecast period, combined with hydrological models, which has become an important development direction to

${ }^{1}$ Leizhi Wang, State Key laboratory of Hydrology, Water Resources and Hydraulic Engineering \& Science, Nanjing Hydraulic Research Institute, Nanjing, Jiangsu, China; E-mail: leizhi668@foxmail.com. 
improve the level of hydrological forecasting [2]. However, affected by factors such as multi-scale complex weather systems and topographical fluctuations, the precipitation presents variation characteristics with complex and high-frequency temporal and spatial changes, which is one of the most difficult meteorological variables to predict in the ensemble numerical forecast system.

At present, the calculation grid of the global ensemble numerical forecast model is relatively coarse, leading to the low spatial resolution of its precipitation forecast information, which does not match the spatial scale of the hydrological forecast model. In addition, the precipitation forecast results are affected by factors such as initial values and model errors [3], so there are still notable quantitative errors and uncertainties, which restrict its application in hydrological forecasting [4]. Therefore, post-processing measures from a statistical perspective such as continuous verification of the temporal and spatial accuracy and error characteristics of numerical precipitation, spatial downscaling, error correction, and probability forecasting are indispensable for obtaining high-resolution, high-precision and reliable ensemble precipitation forecast information. Scholars at home and abroad have carried out a lot of exploration about these two aspects, and actively promoted the improvement of the precipitation forecast performance of the ensemble numerical model. The research and current situation are timely and systematically integrated. Considering the needs of hydrological forecasting applications and analyzing research deficiencies, they propose the future development directions, which is important for mining the availability of numerical forecast precipitation information, and promoting the research and operational application of meteorological and hydrological coupled forecasting.

\section{Development of Ensemble Numerical Forecast and Verification of Precipitation Forecast Accuracy}

The meteorological ensemble numerical forecast system originated in the 1970s and has gone through three development stages of theoretical system improvement, operational system research and development, and weather forecast application. Through disturbing initial values and models, it mainly solves the forecast uncertainty problems caused by initial values and model errors [5]. The long-term disturbance research is mainly carried out in the initial field. The realization method has gradually developed from a simple random disturbance method based on analyzing the error probability distribution to a dynamic disturbance method combining data assimilation and error analysis. For example, the ensemble transform Kalman filter has been widely used [6]. The research on mode disturbance is relatively weak, mainly including single-mode physical parameterization disturbance and multi-mode integration [7] Among them, in terms of multi-mode integration, the most influential one is the TIGGE (THORPEX Interactive Grand Global Ensemble) plan, which integrates the multi-member, multi-element, and multi-time-effect forecast information of the mid-term ensemble forecast system of multiple meteorological service centers [8]. The TIGGE can consider the uncertainty caused by model structure and data assimilation in addition to the initial value error. The implementation of this plan has greatly promoted the verification and interpretation of ensemble forecast products.

A comprehensive understanding of the performance of the ensemble forecast model and the causes of model errors are very important for improving and perfecting the forecast model, and it can also provide a scientific basis for users to select ensemble 
forecast products reasonably. The academic circles have extensively carried out verification of precipitation forecast accuracy for different ensemble models [9-12], which mainly involve two parts of the deterministic accuracy and the probabilistic accuracy. The assessment objects of deterministic accuracy are the ensemble average precipitation or single-member forecast precipitation, related to three aspects of the classification and identification ability of precipitation events with different thresholds, the spatial-temporal quantitative error, and the spatial-temporal dependence structure. The probabilistic verification focuses on the reliability, resolution, forecasting skills and dispersion degree to measure the accuracy of probability forecasting [13]. The study found that the ensemble forecast precipitation generally has problems of light rain nothing-happened reports, heavy rain missed reports, and light rain forecasting techniques not as good as heavy rains. At the same time, there are common problems such as low resolution and problems with under-dispersion or over-dispersion. However, the specific performance of different models varies with model structure, data assimilation, initial value disturbance, physical parameter schemes, etc. The precipitation forecast accuracy of the same model varies with geographic location, climatic characteristics, precipitation characteristics (type, intensity), and forecast period. In addition, the accuracy is also closely related to the time-space scale [9-10]. As shown in figure 1, the MMEF (Multi-model ensemble forecast) object of the three modes of ECMWF, CMA and NCEP to control the forecast precipitation gives the precipitation forecast accuracy of different forecast periods and different spatial locations in the Han River Basin, which has significant The characteristics of temporal and spatial heterogeneity. In general, the current inspections of ensemble precipitation forecasts mostly focus on the deterministic accuracy. Further research should be carried out on the uncertain features such as resolution, forecasting skills, and degree of dispersion.
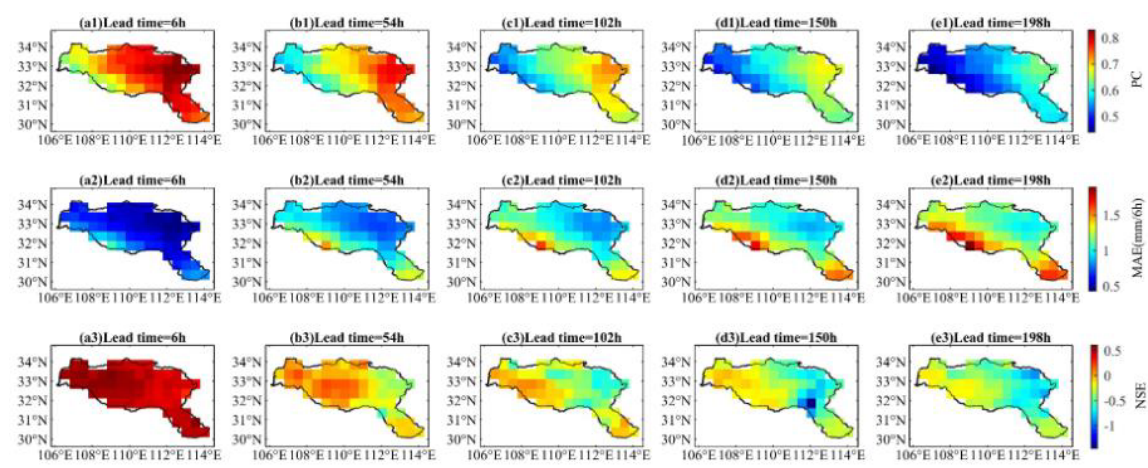

Figure 1. The spatial distribution of the accuracy of the precipitation time series of the multi-model ensemble average forecast in the Han River Basin.

However, the deterministic and probabilistic accuracy verification both rely on reliable ground benchmark precipitation data. The benchmark data used in the existing research mainly includes precipitation observed by discrete rain gauge [12], precipitation retrieval by satellites such as TRMM (The Tropical Rainfall Measuring Mission) [10], CMORPH (the Climate Prediction Center morphing method) and rain gauge fusion precipitation [14]. The spatial coverage and representativeness of precipitation observed by rain gauges may be insufficient. The precipitation of satellite 
grids and other grids have prominent problems of their own errors [15-16], which all affect the reliability of the verification results. Therefore, the establishment of high-quality surface benchmark rainfall data is very important for the verification of ensemble forecast precipitation accuracy, however, it has not yet attracted enough attention. Of course, some scholars have studied accuracy evaluation methods that do not rely on benchmark data. For example, the Extended Triple collocation (ETC) method proposed by McColl et al. [17] relieves the constraint of benchmark precipitation, in which only three sets of independent simulation models or forecast data can be used to evaluate RMSE and $\mathrm{CC}$ but the probabilistic accuracy is not covered.

\section{Statistical Post-processing of Ensemble Forecast Precipitation Statistics and Its Benefits Evaluation}

\subsection{Statistical Post-processing Method}

The statistics post-processing of ensemble forecast precipitation mainly includes three aspects of statistical downscaling, correction of quantitative error and probability forecasting. Scholars at home and abroad have proposed a series of statistical analysis methods, showing a development trend from single function to multi-functional integration. Traditional methods such as EMOS (Ensemble model output statistics), BMA (Bayesian model averaging) and AM (Analog method) have been developed with functions of downscaling, error reduction and probability forecasting. In these methods, the statistical downscaling is generally implied by simple spatial interpolation (such as linear interpolation, neighborhood moving weighted average, etc.) in the matching process of measured precipitation and forecast precipitation [18-20]. The error correction and probability forecasting are the core parts of statistical post-processing.

According to the precipitation probability theoretical distribution involved, the existing statistical post-processing methods are divided into two categories. Methods non-involved to probability distribution, such as multi-mode integration [21], cumulative experience frequency matching correction [22], and AM [18] are the most representative methods. These kinds of methods can avoid the estimation of the precipitation probability distribution, but it is often limited by the lack of sufficient sample data, especially for extreme precipitation, which affects the reliability of the results. The application of second-precipitation forecast products alleviates this problem above to a certain extent $[19,23]$. The method based on the theoretical probability distribution mainly uses the ensemble forecast information to construct the precipitation probability distribution, which can realize the extrapolation of extreme precipitation.

However, due to the large number of zero values and significant bias-normal characteristics in precipitation on certain time scales (especially daily, hour and below), it is usually necessary to select a suitable probability distribution linear and make some proper mathematical processing. The BMA method uses Logistic and Gamma distributions to construct a mixed probability distribution of precipitation probability and non-zero precipitation, and uses the Bayesian weighted average principle to correct the probability of precipitation [23]. The EMOS method uses certain mathematical processing on the single probability distribution, as the theoretical probability distribution linear of the measured precipitation, which establishes the regression 
relationship between the distribution parameters and the ensemble forecast precipitation statistics, so as to obtain the conditional probability distribution of the real precipitation. There are many mathematical processing methods for the linear probability distribution. For example, Scheuerer [24] censors the part of the generalized extreme value distribution less than zero, and Scheuerer and Hamill [20] censor the part of the Gamma distribution less than zero and shift it to the left. In addition to the above-mentioned methods involving univariate probability distribution estimation, the Bayesian joint probability (BJP) distribution [25-26] and the meta-Gaussian distribution [27] for joint forecasting and other multivariate probability methods for measured precipitation, are applied to statistical post-processing, thus the correlation between forecast and measured precipitation are taken into consideration.

In general, the existing statistical post-processing methods have shown positive effects in correcting quantitative errors and improving forecasting skills. However, most methods take the forecast precipitation of a single time period and isolated grid points (or stations) as the research object, which often destroys the temporal and spatial continuity of precipitation $[25,26,28]$. For example, BMA and EMOS methods can directly derive the conditional probability distribution of forecast precipitation at each grid point and time period. If ensemble forecast precipitation data is required, it must be randomly sampled from the precipitation probability distribution at each grid point. Obviously, this way disturbs the temporal and spatial dependency structure of precipitation between adjacent grid points and adjacent periods [29]. How to reconstruct the temporal and spatial dependence of forecast precipitation is a major problem in post-statistical processing? The existing studies have adopted the rank order structure based on a specific multivariate dependent template to re-ordered the above-mentioned post-processing methods after outputting ensemble forecast precipitation, to achieve spatial correlation and temporal continuity reconstruction, specifically including two types of empirical method and parameterization method.

Among the empirical methods, the Schaake shuffle method [29] has been widely used. It is a traditional method that uses the temporal and spatial rank relationship of historically similar measured precipitation as a template [27,30], but more complex statistics dependency relation cannot be considered. It also ignores the particularity of the atmospheric state and evolution trend during the forecast period [31]. Scheuerer et al. [30] considered the similarity between historical precipitation and the marginal distribution of precipitation during the forecast period, and improved the selection method of historical dates in the Schaake shuffle method. Ensemble copula coupling (ECC) is also an empirical method. It uses the numerical model to output the temporal and spatial continuous structure contained in the ensemble forecast precipitation as a template, and the best possible result is only to recover the model precipitation structure [32]. The parameterization method also uses the temporal and spatial dependence of historically similar measured precipitation as a template to construct a multivariate joint probability distribution of precipitation at adjacent grid points and periods within a certain temporal and spatial range, which is suitable for low-dimensional temporal and spatial scales. The connection function among variables can be Gaussian copula etc. [33].

Some scholars have also combined the method of geo-statistics output disturbance with the method of ensemble model output statistics to explore the reconstruction of the spatial structure of precipitation [34]. Lerch et al. [28] compares the effects of Schaake shuffle, ECC, and Gaussian copula methods. It is found that Schaake shuffle is generally better than Copula methods. However, the effectiveness of various methods 
largely depends on the degree of fidelity of their own structures of the adopted models of forecast precipitation or historically similar precipitation. In general, the research on this issue is still in its infancy. A reasonable precipitation spatio-temporal dependence structure is very important for hydrological forecasting [30, 35]. Therefore, it is urgent to develop a method that can improve spatial resolution and reduce time and space. A statistical post-processing method for improving spatial resolution, reducing temporal-spatial errors and reasonable reconstruction of temporal and spatial dependent structures is urgent to be developed.

\subsection{Effect Evaluation of Statistical Post-Processing}

Effect evaluation is an important way to understand the performance of statistical post-processing methods and provide feedback information for method improvement. Comparative studies on the effects of different post-processing methods have been extensively carried out, focusing on the analysis of the improvement benefits of the deterministic and probabilistic accuracy of ensemble precipitation. The article adopts the coupling method of Quantile-Mapping (QM) and Joint Probability Distribution with Censored Data (CJPD) to carry out the error correction study of precipitation forecast in the Han River Basin. It is found that regardless of the forecast periods, the improvement benefits of the PC, MAE and NSE indicators all exceed 10\%, and present a spatiotemporal pattern in which the western part of the basin is higher than the eastern part, and this divergence increases significantly with the extension of the forecast periods, which is highly consistent with the temporal and spatial distribution characteristics of the forecast precipitation PC before the correction (figure 2). Zhao et al. [36] compared the frequency matching correction method with the BJP method and found that both can significantly reduce the quantitative error, but the former cannot improve the dispersion of the model forecast precipitation, and the latter can improve the reliability and consistency of the forecast precipitation. At the same time, it is recognized that the improvement benefit is related to the correlation coefficient between forecast and actual precipitation. Medina et al. [37] found that the AM method is better than the logistic regression method in improving forecasting techniques, reliability and resolution of ECMWF (European Centre for Medium-Range Weather Forecasts) and GEFS (Global Ensemble Forecast System) in ensemble numerical forecast system. The improvement benefits of GEFS precipitation with precipitation forecasting skills less than 0 are very obvious. However, Scheuerer and Hamill [20] pointed out that the AM method is not as effective as the EMOS method based on censored Gamma distribution in improving the probabilistic accuracy of GEFS precipitation.

It can be seen that there are differences in the performance of different methods. The benefits of statistical post-processing are related to the quality of the precipitation forecast by the original model. In addition, some scholars have found that the effect of improving the accuracy of forecasting precipitation varies with precipitation magnitude and temporal and spatial location. Stauffer et al. [38] found that the extent of improving the BSS (Brier score skill) of the EMOS method tends to decrease with the prolongation of the forecast period. At the same time, the benefit of improving the forecasting skills of light rain is more obvious than that of heavy rain.

Verkade et al. [31] observed that statistical post-processing methods cannot improve the reliability and resolution of ensemble precipitation forecasts at the same time. The improvement of the reliability item CRPSS $_{\text {rel }}$ in CRPSS (Continuous ranked 
probability score skill) is at the cost of the loss of the resolution item CRPSS res $_{\text {. The }}$ improvement of CRPSS ${ }_{\text {rel }}$ gradually decreases with the increase of precipitation. In general, the research on systematically evaluating the certainty and probabilistic accuracy gains of ensemble precipitation, on identifying its temporal and spatial distribution characteristics and its influencing factors is not sufficient. In addition, the quantitative analysis of the temporal and spatial dependent structural changes of precipitation is relatively weak.

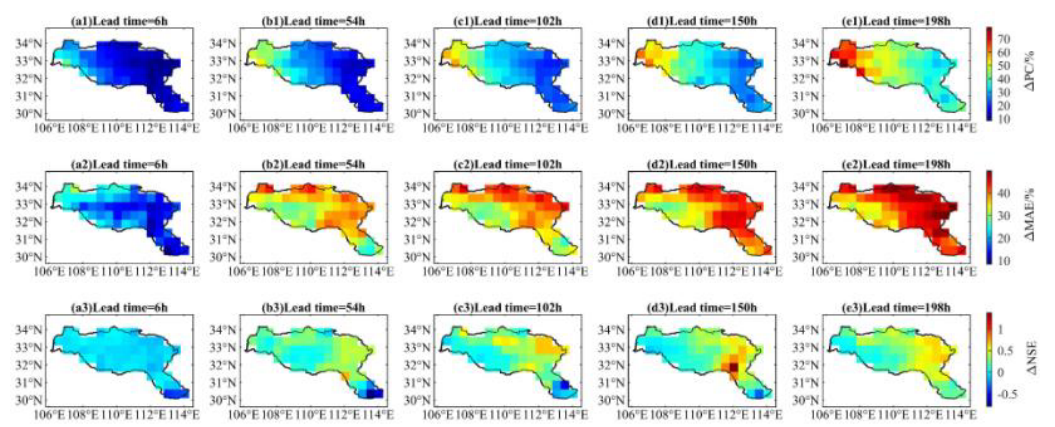

Figure 2. The spatial distribution of the accuracy gains of the raster forecast precipitation in the Han River Basin after the QM-CJPD method is corrected.

\section{Shortcomings and Prospects}

In recent years, a large number of studies have been carried out on the precipitation accuracy and statistical post-processing of ensemble forecasts. It has been confirmed that statistical post-processing has shown positive effects in solving low spatial resolution, improving certainty and probabilistic accuracy, which brings new opportunities to improve the level of hydrological forecasting. However, the existing statistical post-processing methods still have some shortcomings in the reconstruction of the temporal and spatial dependence structure of precipitation, in the identification of the temporal and spatial heterogeneity of the forecast precipitation accuracy gains, which is the focus of future research.

Regarding the accuracy verification of the precipitation information forecast in the ensemble numerical forecast system, while analyzing the deterministic accuracy, it is also necessary to focus on the uncertainty characteristics such as the resolution, the forecasting skills, and the degree of dispersion, so as to provide more detailed prior knowledge for statistical post-processing.

The mainstream statistical post-processing methods mostly focus on forecast precipitation in isolated temporal and spatial locations as the research object, which often destroys the temporal and spatial dependency structure of forecast precipitation. Research on this problem is still very weak, and it is urgent to develop a statistics post-processing method for ensemble forecast precipitation that integrates improving spatial resolution, correcting quantitative errors, and reconstructing spatio-temporal dependent structures.

The verification of the ensemble numerical model precipitation forecast and the performance of statistical post-processing methods are still needed to be vigorously deepened. First, the lack of spatial coverage, representativeness, and accuracy of 
surface benchmark data may affect the objective evaluation of ensemble precipitation accuracy and post-processing methods. It is urgent to develop spatially continuous and reliable ground benchmark precipitation as the base. Second, the systematic research on the temporal and spatial distribution characteristics and influencing factors of the statistical post-processing to improve the certainty and the probabilistic accuracy gains of ensemble precipitation is relatively weak. At the same time, the quantitative analysis of the influence of the temporal and spatial dependence of precipitation is obviously lacking.

\section{Acknowledgments}

National Key R\&D Program of China(2016YFC0400902); National Science Foundation of China (52009081); Special funded project for basic scientific research operation expenses of the Central Public Welfare Scientific Research Institutes of China (Y519006).

\section{References}

[1] Xia J, Wang HY, Gan YY, et al. Research progress in forecasting methods of rainstorm and flood disaster in China. Torrential Rain and Disasters. 2019 Otc; 38(5): 416-421. (In Chinese)

[2] Zhao LN, Liu Y, Dang HF, et al. The progress on application of ensemble prediction to flood forecasting. Journal of Applied Meteorological Science. 2014 Dec; 25(6): 641-653. (In Chinese)

[3] Chang J, Peng XD, Fan GZ, et al. Error Calibration of Numerical Weather Prediction with Historical Data. Acta Meteorologica Sinica.2015 Apr; 73(2): 341-354. (In Chinese)

[4] Cloke HL, Pappenberger F. Ensemble flood forecasting: A review. Journal of hydrology. 2009 Sep; 375(3-4): 613-626.

[5] Chen J, Chen DH, Yan H. A Brief review on the development of ensemble prediction system. Journal of Applied Meteorological Science. 2002 Apr; 13(4): 497-507. (In Chinese)

[6] Gao L, Chen J, Zheng JW, et al. Progress in researches on ensemble forecasting of extreme weather based on numerical models. Advances in Earth Science. 2019 Jun; 34(7): 706-716. (In Chinese)

[7] Duan MJ, Wang P X. Advances in researches and applications of ensemble prediction. Transactions of Atmospheric Sciences. 2004 Feb; (2): 279-288. (In Chinese)

[8] Park YY, Buizza R, Leutbecher M. TIGGE. Preliminary results on comparing and combining ensembles. Quarterly Journal of the Royal Meteorological Society. 2008 Jan; 134(637): 2029-2050.

[9] Zhao LN, Wu H, Tian FY. Assessment of probabilistic precipitation forecasts for the huaihe basin using TIGGE data. Meteorological Monthly. 2010 Jun; 36(7): 133-142. (In Chinese)

[10] Su X, Yuan H, Zhu Y, et al. Evaluation of TIGGE ensemble predictions of Northern Hemisphere summer precipitation during 2008-2012. Journal of Geophysical Research: Atmospheres. 2014 Feb; 119(12): 7292-7310.

[11] Louvet S, Sultan B, Janicot S, et al. Evaluation of TIGGE precipitation forecasts over West Africa at intraseasonal timescale. Climate Dynamics. $2016 \mathrm{Jul}$; 47(1-2): 31-47.

[12] Aminyavari S, Saghafian B, Delavar M . Evaluation of TIGGE ensemble forecasts of precipitation in distinct climate regions in Iran. Advances in Atmospheric Sciences. 2018 Feb; 35(4): 457-468.

[13] Bi BG, Dai K, Wang Y, et al. Advances in techniques of quantitative precipitation forecast. Journal of Applied Meteorological Science. 2016 Sep; 27(5): 534-549.(In Chinese)

[14] Zhi XF, Lv Y. Calibration of the multimodel precipitation forecasts in China using the frequency matching method. Transactions of Atmospheric Sciences. 2019 Sep; 42(6): 814-823. (In Chinese)

[15] Tian Y, Peters-Lidard CD, Eylander JB, et al. Component analysis of errors in satellite-based precipitation estimates. Journal of Geophysical Research: Atmospheres. 2009 Dec; 114(D24).

[16] Shen Y, Zhao P, Pan Y, et al. A high spatiotemporal gauge-satellite merged precipitation analysis over China. Journal of Geophysical Research: Atmospheres. 2014 Mar; 119(6): 3063-3075.

[17] McColl KA, Vogelzang J, Konings AG, et al. Extended triple collocation: Estimating errors and correlation coefficients with respect to an unknown target. Geophysical Research Letters. 2014 Aug; 41(17): 6229-6236. 
[18] Hamill TM, Whitaker JS. Probabilistic quantitative precipitation forecasts based on reforecast analogs: Theory and application. Monthly Weather Review. 2006 Nov; 134(11): 3209-3229.

[19] Hamill TM, Scheuerer M, Bates GT. Analog probabilistic precipitation forecasts using GEFS reforecasts and climatology-calibrated precipitation analyses. Monthly Weather Review. 2015 Aug; 143(8): 3300-3309.

[20] Scheuerer M, Hamill TM. Statistical postprocessing of ensemble precipitation forecasts by fitting censored, shifted gamma distributions. Monthly Weather Review. 2015a Nov; 143(11): 4578-4596.

[21] Cane D , Milelli M . Multimodel SuperEnsemble technique for quantitative precipitation forecasts in Piemonte region. Natural Hazards \& Earth System Sciences. 2010 Feb; 10(2): 265-273.

[22] $\mathrm{Li} \mathrm{J}, \mathrm{Du} \mathrm{J}$, Chen CJ. Introduction and analysis to frequency or area matching method applied to precipitation forecast bias correction. Meteorological Monthly. 2014 Mar; 40(5): 580-588. (In Chinese)

[23] Raftery AE, Gneiting T, Balabdaoui F, et al. Using Bayesian model averaging to calibrate forecast ensembles. Monthly weather review. 2005 May; 133(5): 1155-1174.

[24] Scheuerer M. Probabilistic quantitative precipitation forecasting using ensemble model output statistics. Quarterly Journal of the Royal Meteorological Society. 2013 Jul; 140(680): 1086-1096.

[25] Robertson DE, Shrestha DL, Wang QJ. Post processing rainfall forecasts from numerical weather prediction models for short term streamflow forecasting. Hydrology \& Earth System Sciences Discussions. 2013 Sep; 17(9): 3587-3603.

[26] Shrestha DL, Robertson DE, Bennett J C, et al. Improving precipitation forecasts by generating ensembles through postprocessing. Monthly Weather Review. 2015 Sep; 143(9): 3642-3663.

[27] Li W, Duan Q, Ye A, et al. An improved meta-Gaussian distribution model for post-processing of precipitation forecasts by censored maximum likelihood estimation. Journal of Hydrology. 2019 Jul; 574: 801-810.

[28] Lerch S, Baran S, Möller A, et al. Simulation-based comparison of multivariate ensemble post-processing methods. Nonlinear Processes in Geophysics Discussions. 2020 Jun: 1-30.

[29] Clark M, Gangopadhyay S, Hay L, et al. The Schaake shuffle: A method for reconstructing space-time variability in forecasted precipitation and temperature fields. Journal of Hydrometeorology. 2004 Feb; 5(1): 243-262.

[30] Scheuerer M, Hamill TM, Whitin B, et al. A method for preferential selection of dates in the Schaake shuffle approach to constructing spatiotemporal forecast fields of temperature and precipitation. Water Resources Research. 2017 Mar; 53(4): 3029-3046.

[31] Verkade JS, Brown JD, Reggiani P, et al. Post-processing ECMWF precipitation and temperature ensemble reforecasts for operational hydrologic forecasting at various spatial scales. Journal of Hydrology. 2013 Sep; 501: 73-91.

[32] Schefzik R, Thorarinsdottir TL, Gneiting T. Uncertainty quantification in complex simulation models using ensemble copula coupling. Statistical Science. 2013 Nov; 28(4): 616-640.

[33] Möller A, Lenkoski A, Thorarinsdottir TL. Multivariate probabilistic forecasting using ensemble Bayesian model averaging and copulas. Quarterly Journal of the Royal Meteorological Society. 2012 Sep, 139(673): 982-991.

[34] Feldmann K, Scheuerer M, Thorarinsdottir TL. Spatial postprocessing of ensemble forecasts for temperature using nonhomogeneous Gaussian regression. Monthly Weather Review. 2015 Mar, 143(3): 955-971.

[35] Bellier J, Bontron G, Zin I. Using meteorological analogues for reordering postprocessed precipitation ensembles in hydrological forecasting. Water Resources Research. 2017 Nov; 53(12): 10085-10107.

[36] Zhao T, Bennett JC, Wang QJ, et al. How suitable is quantile mapping for postprocessing GCM precipitation forecasts?. Journal of Climate. 2017 May; 30(9): 3185-3196.

[37] Medina H, Tian D, Marin FR, et al. Comparing GEFS, ECMWF, and postprocessing methods for ensemble precipitation forecasts over Brazil. Journal of Hydrometeorology. 2019 Apr; 20(4): 773-790.

[38] Stauffer R, Umlauf N, Messner JW, et al. Ensemble postprocessing of daily precipitation sums over complex terrain using censored high-resolution standardized anomalies. Monthly Weather Review. 2017 Mar; 145(3): 955-969. 